\title{
Efecto de un Consorcio de cianobacterias sobre la obtención de biomasa vegetal de la gulupa (Passiflora edulis f. edulis sims) bajo condiciones de campo en el municipio de Marinilla - Antioquia
}

\section{Effect of a Cyanobacteria consortium on obtaining the gulupa's vegetal biomass (Passiflora edulis f. Edulis sims) under field conditions in the municipality of Marinilla - Antioquia}

\author{
Yuli A. Pérez Cardona*, Cindy A. García Salazar, \\ Leonardo A. Ríos Osorio ${ }^{\ddagger \neq}$ ORCID 0000-0002-7127-4629, \\ Luisa M. Múnera Porras ${ }^{\dagger+}$ ORCID 0000-0002-0215-9993
}

\section{Resumen}

Introducción: las cianobacterias poseen la capacidad de fijar nitrógeno, producir vitaminas y aminoácidos que estimulan el crecimiento vegetal, particularmente forman asociaciones con plantas vasculares y no vasculares, actuando como biofertilizantes, y convirtiéndose en una alternativa para disminuir la aplicación de agroquímicos que afectan la calidad de los suelos y la salud humana. Estos microorganismos han venido utilizándose para aumentar la producción en cultivos de interés comercial, aunque la mayoría de las investigaciones se han centrado en el arroz y otros cereales. La gulupa (Passiftora edulis f. edulis Sims), es una fruta originaria de las regiones tropicales, su agradable sabor y propiedades antioxidantes hacen que sea atractiva comercialmente en especial para la exportación, lo que ha incentivado el desarrollo de este cultivo en el país.

Objetivo: el presente trabajo tuvo como objetivo evaluar el efecto de un consorcio de cianobacterias sobre la obtención de biomasa vegetal de gulupa (Passiffora edulis f. edulis Sims.) bajo condiciones de campo.

Métodos: se diseñó un estudio experimental comparativo. Se evaluó el efecto biofertilizante de las cianobacterias con tres tratamientos. El crecimiento de las plantas se midió en términos de longitud de tallo y raíces, peso fresco de planta y raíces, y número de hojas y ramas durante seis meses de siembra.

Resultados y conclusión: los resultados obtenidos indican que existe una respuesta por parte de la planta al aplicar la inoculación con cianobacterias; sin embargo, no se puede establecer cuál de los tratamientos indujo dicha respuesta.

Palabras clave: Agroquímicos, Biofertilizante, Cianobacterias, Consorcio, Cultivo, Gulupa (Passiflora edulis f. edulis Sims).

\footnotetext{
* Estudiantes de Microbiología Industrial y Ambiental, Universidad de Antioquia, Medellín, Colombia.

† Bacteriólogo y Lab. Clínico. Especialista en CBB, Parasitología Humana. Ph.D. en Sostenibilidad. Grupo de investigación Salud y Sostenibilidad, Escuela de Microbiología, Universidad de Antioquia, Medellín, Colombia.

Medellín, Colombia.

†† Microbióloga Industrial y Ambiental, Magister en Biología, Grupo de Investigación Salud y Sostenibilidad, Escuela de Microbiología, Universidad de Antioquia, Medellín, Colombia.

Recepción: 30/01/2020. Aceptación: 23/06/2020

Cómo citar este artículo: Pérez-Cardona YA, García-Salzar CA, Ríos-Osorio LA, Múnera Porras LM. Efecto de un Consorcio de cianobacterias sobre la obtención de biomasa vegetal de la gulupa (Passiflora edulis f. edulis sims) bajo condiciones de campo en el municipio de Marinilla Antioquia. Hechos Microbiol. 2020;11(1-2):12-21. DOI: 10.17533/udea.hm.v11n1a02
} 


\section{Abstract}

Introduction: Cyanobacteria possess the ability to fix nitrogen, produce vitamins and amino acids that stimulate plant growth, form associations with vascular and non-vascular plants, acts as biofertilizers, and as an alternative to the application of agrochemicals, reduce the harmful affect these have on the quality of the soils and human health. In culture of commercial interest, these microorganisms have been used to increase production although most research has focused on rice and other cereals. Gulupa (Passiflora edulis f. edulis Sims), is a fruit native to tropical regions. Its pleasant taste and antioxidant properties make it commercially attractive, especially for export, which has encouraged the development of this culture in the country.

objective: The objective of this work was to evaluate the effect of a consortium of cyanobacteria on obtaining plant biomass from gulupa plants (Passiflora edulis f. edulis Sims.) under field conditions.

Methods: A comparative experimental study was designed. The biofertilizing effect of cyanobacteria was evaluated with three treatments. The growth of the plants was measured in terms of length of stem and roots, fresh weight of plant and roots, and number of leaves and branches during six months of planting.

Results and conclusion: The results obtained indicate that there is a response from the plant when inoculation with cyanobacteria is applied; however, it cannot be established which of the treatments induced that response.

Key words: Agrochemicals, Biofertilizer, Cyanobacteria, Consortium, Culture, Gulupa (Passiflora edulis F. edulis Sims).

\section{Introducción}

El uso de agroquímicos en los suelos ha incrementado la capacidad productiva de los agroecosistemas, aunque ha provocado consecuencias no favorables, como el aumento en la contaminación ambiental y la degradación de los recursos suelo y agua. ${ }^{1}$ Además, podría ejercer efectos nocivos sobre las poblaciones microbianas presentes, ${ }^{2}$ así como producir afecciones a la salud humana, como irritación en la piel, asfixia, paro respiratorio, convulsiones y hasta conducir a la muerte..$^{3-4}$ Según la Organización Mundial de la Salud (OMS) se estima que cada año se producen 25 millones de intoxicaciones por venenos agroquímicos en el mundo, y alrededor de 20.000 muertes. ${ }^{5}$

La necesidad de los productores agrícolas de desarrollar al máximo su producción y mejorar la calidad de las cosechas, sumado al elevado costo de la fertilización química, presentan a la biofertilización como una alternativa útil que puede complementar al sistema productivo, ${ }^{6}$ contrarrestando los efectos negativos generados por el uso de agroquímicos. ${ }^{7}$ Entre los microorganismos más utilizados para la formulación de los inoculantes se destacan los géneros bacterianos de Bacillus, Pseudomonas, Azospirillum, Azotobacter, Rhizobium, Bradyrbizobium, Sinorbizobium, Mesorbizobium y Streptomyces. Estos microorganismos habitan la rizósfera y promueven el crecimiento de las plantas, por tal razón se los denomina PGPR (Plant Growth Promoting Rhizobacteria $)^{1}$ o conocidas como rizobacterias promotoras de crecimiento vegetal, en español. De la misma forma, el uso de cianobacterias presenta múltiples ventajas, pues son capaces de producir reguladores de crecimiento como auxinas tipo ácido indolacético, ${ }^{8}$ citoquininas $^{9}$ y giberelinas ${ }^{10}$, así mismo de aumentar la disponibilidad de fósforo por medio de la emisión de ácidos orgánicos, ${ }^{11}$ la producción de fosfatasas extracelulares, ${ }^{12}$ y algunas de fijar nitrógeno. Producen una amplia variedad de metabolitos secundarios con actividad antiviral, antibacterial y antifúngica. ${ }^{13-14}$

Los principales estudios usando cianobacterias como PGPR se han realizado en cultivos de arroz. Uno de estos estudios indica que la inoculación con cianobacterias condujo a una mayor longitud de las plantas en $15,9 \%$, mientras que la biomasa vegetal (base en peso seco) aumentó en 27,3 \%, y un incremento en el peso fresco del 75,36 \%, ${ }^{15-16}$ en este estudio se reportó que las cianobacterias Anabaena vaginicola ISC90 y Nostoc calcicola SC89, aisladas de suelos arroceros de Irán, promovieron el crecimiento de otras plantas como el pepino (Cucumis sativus), tomate (Solanum lycopersicum) y calabaza (Cucurbita maxima), lo cual evidencia las diversas aplicaciones de las cianobacterias como promotoras de crecimiento vegetal en diferentes tipos de cultivos. ${ }^{17}$

La gulupa (Passiflora edulis f. edulis Sims) es una de las especies del género Passiflora, conocida en Colombia 
como maracuyá morado y en la zona andina como gulupa, se cultiva comercialmente en regiones tropicales y subtropicales. Es fuente de carbohidratos, calorías, proteínas, minerales y vitaminas (A, B3, B12 y C); además, las características nutricionales de la pulpa y el néctar la destacan con altos contenidos de fenoles totales y alta capacidad antioxidante, sugiriendo que el consumo de esta fruta puede contribuir al beneficio de la salud humana lo que la hace más atractiva para la exportación. ${ }^{18}$

A nivel mundial los estudios de biofertilización con cianobacterias se han centrado en cultivos de cereales, leguminosas y especias; ${ }^{15-19-20}$ sin embargo, no se han explorado estas propiedades en plantas frutales, concretamente en pasifloras.

De acuerdo con lo anterior, esta investigación se propuso evaluar el efecto de un consorcio de cianobacterias sobre la obtención de biomasa vegetal de gulupa (Passiflora edulis f. edulis Sims.) bajo condiciones de campo.

\section{Materiales y métodos}

\section{TIPO DE ESTUDIO}

Se diseñó un estudio experimental comparativo completamente aleatorio. El ensayo incluyó tres tratamientos cada uno con 12 réplicas, para un total de 108 unidades experimentales. La distribución de las unidades experimentales fue al azar a través de los 4 surcos de siembra del terreno.

\section{Obtención del Consorcio de Cianobacterias}

Las muestras de agua con el consorcio de cianobacterias se tomaron a la entrada del río Nare al embalse Peñol-Guatapé, donde hay presencia de un "bloom" de cianobacterias evidenciado por su coloración. El embalse se encuentra a una altura aproximada de 1887,5 m.s.n.m, una temperatura media de $20^{\circ} \mathrm{C}$, y cubre un área de 6.240 ha, con un tiempo de retención hidráulico de 285 días. $^{21,22}$ Estudios anteriores reportan la presencia de las cianobacterias Microcystis aeruginosa, Synechococcus rubescens y Cyanobium gracile como parte del consorcio.23 Las muestras fueron recolectadas por medio de una malla de fitoplancton (Turtox Bioweb, COL), con un tamaño de poro de $10 \mu \mathrm{m}$. El arrastre con la malla se realizó a una velocidad promedio de $500 \mathrm{rpm}$ durante 5 min. Estas se almacenaron en recipientes plásticos de policarbonato estériles tapa rosca de $1000 \mathrm{~mL}$; posteriormente fueron transportadas en neveras plásticas a una temperatura entre 2 y $8{ }^{\circ} \mathrm{C}$, hasta la finca donde se inocularon las plántulas de gulupa.

Adicionalmente, se realizó la determinación de parámetros fisicoquímicos como $\mathrm{pH}$, temperatura, oxígeno disuelto, conductividad y zona fótica de la ubicación en la cual se obtuvo el consorcio de cianobacterias.

\section{Establecimiento del cultivo de gulupa (Passiflora EDULIS F. EDULIS SIMS)}

El estudio se llevó a cabo en una finca con manejo orgánico ubicada en la vereda El Chagualo del municipio de Marinilla, Antioquia, situada en la región centro andina colombiana, con una altitud de 2133 m.s.n.m. La zona corresponde según la clasificación de Holdridge a un bosque húmedo premontano (bh-PM), posee una temperatura promedio de $17^{\circ} \mathrm{C}$, con régimen de lluvias entre 1000 y $4000 \mathrm{~mm}$ por año y una humedad relativa de $80 \% .^{24}$

Las plántulas para el estudio fueron obtenidas en un vivero con 50 días de crecimiento, se excluyeron aquellas que presentaron deterioro como manchas foliares, antracnosis, alternariosis o señales de ataques por fitopatógenos, que resultan en cambios adversos en la forma, función o integridad de la planta, interfiriendo con la formación, translocación o utilización de nutrientes minerales y agua. Para las siembras se realizaron hoyos con paladraga de aproximadamente $10 \mathrm{~cm}$ de profundidad con una distancia de $25 \mathrm{~cm}$ entre cada hoyo. Los cuatro surcos distaban entre sí $60 \mathrm{~cm}$ con una extensión de $5 \mathrm{~m}$; en cada uno de los surcos se sembraron 27 plantas, para un total de 108 plantas, distribuidas entre los tres tratamientos. La asignación de cada una de las plantas a los diferentes tratamientos se hizo al azar usando el programa Excel con la función de asignación de números aleatorios.

\section{INOCULACIÓN DE LOS CULTIVOS DE GULUPA CON CIANOBACTERIAS}

Para precisar el efecto de las cianobacterias en el crecimiento de las plantas de gulupa sembradas en campo se distribuyó el estudio en tres tratamientos, 36 plantas en cada tratamiento. El tratamiento 1 (T1) constituyó el control negativo del estudio, el cual no se inoculó con las cianobacterias; el tratamiento 2 (T2) contenía 
las plántulas de gulupa cuyas raíces se sumergieron durante 5 minutos en la muestra de agua que contenía el consorcio de cianobacterias, acto seguido se inoculó el suelo donde se sembró la plántula con $100 \mathrm{~mL}$ de la misma muestra; las plántulas fueron re-inoculadas en 3 ocasiones, con un intervalo de tiempo de 15 días. El tratamiento 3 (T3) se realizó similar al T2, pero con adición a cada planta de 300 g de compost orgánico.

\section{Medición de LAS VARIABLES}

Las mediciones se efectuaron cada 15 días, durante 6 meses. En cada medición se retiraron tres plantas de cada tratamiento, las cuales fueron extraídas del suelo con la ayuda de un palustre, teniendo especial cuidado en no dañar las raíces, luego se realizó un lavado con agua para retirar el exceso de suelo, se secaron con papel absorbente y se efectuaron las mediciones, que constituyen cada una de las variables evaluadas. La longitud de la planta (LP) se midió desde el meristema apical hasta donde comienza la raíz, la longitud de la raíz (LR) se midió desde donde termina el cuello del tallo hasta la cofia de la misma. Estas mediciones se realizaron con cinta métrica. Para el peso fresco de la planta (PFP) y de la raíz (PFR) se cortó el cuello del tallo, donde comienza la raíz con la ayuda de un bisturí y se pesaron por separado (raíz y planta) en balanza gramera digital (HAND HELD). Para las variables número de ramas (NR) y número de hojas $(\mathrm{NH})$ se efectuó un conteo visual de cada planta.

Al finalizar cada procedimiento, se registraron manualmente los datos de las diferentes variables, además de la temperatura y humedad relativa del lugar.

\section{Plan de análisis}

Los datos obtenidos de cada medición fueron depositados en una base de datos en Excel, para después ser analizados en el software SPSS-IBM versión $25^{\circledR}$. En todos los análisis se tomó una significación estadística de $\mathrm{p} \leq 0,05$.

Se realizaron estadísticos descriptivos de las variables en los tres tratamientos para definir cuál fue la distribución y el comportamiento de estas a través del tiempo, por medio de medidas de tendencia central y dispersión. Se determinó la correlación de las variables de respuesta en el tiempo por medio del coeficiente de Spearman, dado que los datos no siguieron una distribución normal, y para describir el efecto simultáneo de los tratamientos sobre las variables de respuesta a través del tiempo se realizó un modelo de regresión lineal. Con el fin de precisar cuál de los tratamientos tuvo un efecto diferencial frente a los demás se utilizó, la prueba no paramétrica de Kruskal-Wallis.

\section{Resultados}

Los resultados presentados corresponden al análisis de los datos obtenidos, a partir de la medición de las plantas cada 15 días durante seis meses.

\section{EsTADÍSTICOS DESCRIPTIVOS}

Al examinar los promedios de las variables designadas para evaluar el crecimiento de las plantas, se observó un comportamiento uniforme de los tratamientos en el transcurso del tiempo (Fig. 1).

La tendencia de los datos de la variable LP mostró mayor similitud en el comportamiento de T1 y T3; la aplicación del consorcio de cianobacterias y el abono orgánico (T3) presentó una longitud de la planta de $17,087 \mathrm{~cm}$, siendo este el promedio más alto respecto a los otros tratamientos (Fig. 1A).

Para la variable LR (Fig. 1B) se observó que los tratamientos presentaron un comportamiento similar al rededor de los 102 días; sin embargo, los datos estuvieron muy dispersos durante el tiempo de evaluación. No obstante, T1 fue el que mejor desarrollo de la raíz con un promedio de $7,17 \mathrm{~cm}$ de crecimiento, seguido de T3 con 6,7 cm de longitud.

La variable PFP reveló promedios cercanos para T1 y T3 de 0,1 g y 0,9 g respectivamente, indicando un aumento del peso fresco de la planta para ambos tratamientos (Fig. 1C). Los datos de la variable PFR demostraron un comportamiento cercano entre los tres tratamientos con un promedio superior de T3 de 0,44 g y T1 de 0,37 g (Fig. 1D). 

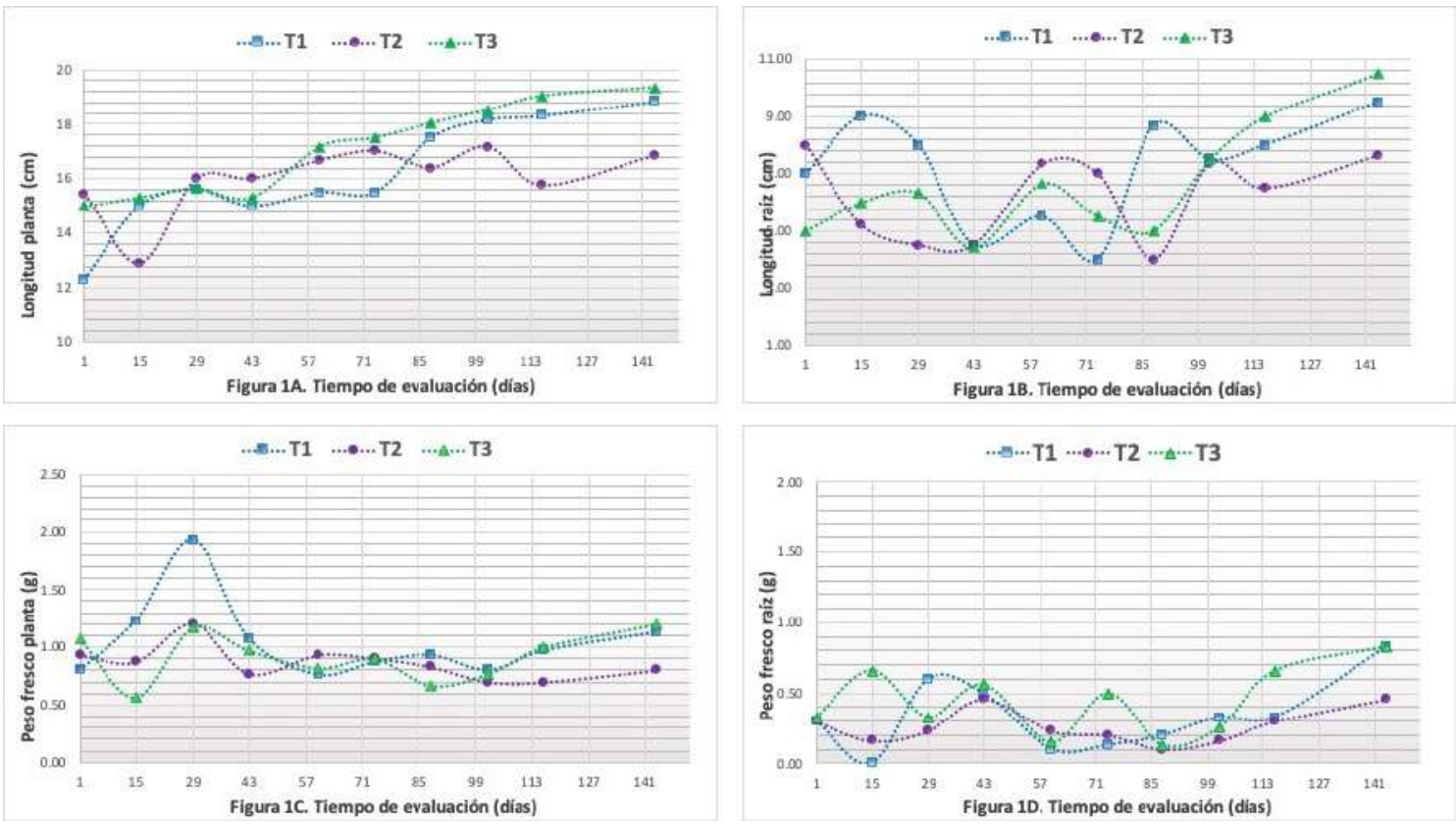

Figura 1. Promedios de las variables de crecimiento de la gulupa evaluadas en el tiempo para cada tratamiento

\section{Correlaciones}

Después de realizar la prueba de normalidad con un $p>0,05$ se estableció que los datos no siguieron una distribución normal, por tanto, se determinó el comportamiento de las variables con el coeficiente de correlación de Spearman y un modelo de regresión lineal.

La variable LP presentó una correlación positiva al obtenerse un Rho 0,681 sugiriendo que la planta tuvo un incremento en el transcurso del tiempo de muestreo en cuanto al coeficiente de determinación $\mathrm{R}^{2}$ de 0,467 (Fig. 2), lo que indica que posiblemente el aumento de la longitud de la planta no tuvo una relación estrictamente lineal al tiempo de evaluación. La variable LR, en función del coeficiente de correlación de Spearman y el coeficiente de determinación manifestaron valores bajos, con un Rho de 0,385 lo cual representa una correlación negativa, y plantea que el aumento de la longitud de la raíz no fue significativo debido al tiempo, adicionalmente un $\mathrm{R}^{2}$ 0,179, que denota baja linealidad de los datos a través del tiempo. La variable PFP señala una correlación de Spearman nula con un Rho -0,096 y un coeficiente de determinación bajo $\mathrm{R}^{2}$ 0,013, lo que explica que hubo una tendencia a disminuir en el peso fresco de la planta, respecto al tiempo de evaluación. De igual manera, la variable PFR evidenció que la raíz no ganó peso representativo en el tiempo, ni tuvo linealidad dado que los valores de Rho y de $\mathrm{R}^{2}$ fueron 0,253 y 0,120 respectivamente.

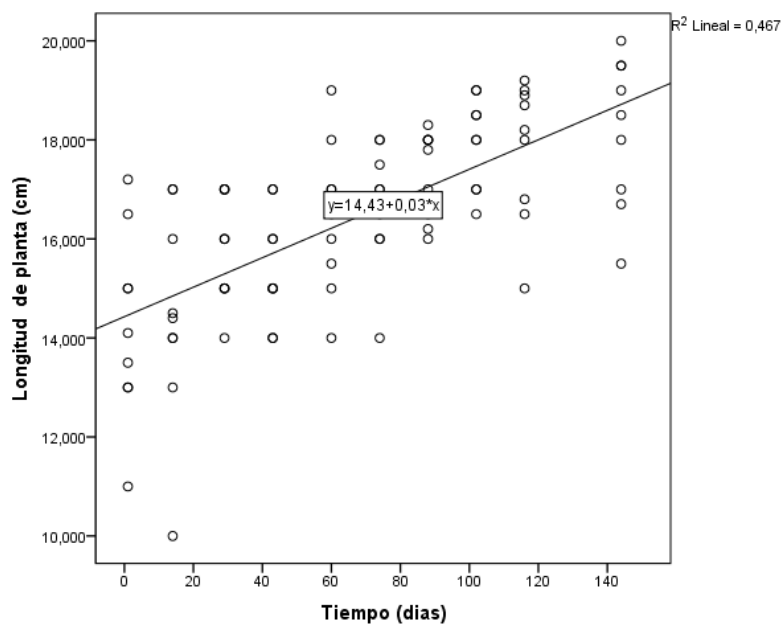

Figura 2. Gráfico de regresión lineal para la variable (LP) en el tiempo 
La prueba H de Kruskal Wallis, se hizo para concluir si los tratamientos aplicados tuvieron algún efecto sobre el crecimiento de las plantas, respecto a las variables de respuesta evaluadas en el tiempo.

Los resultados de esta prueba determinaron que no hubo diferencias significativas al inocular las plantas de gulupa con cianobacterias y que la variable LP fue la única que generó un valor de significancia $(\mathrm{p}=0,05)$ (Fig. 3), pero no se puede diferenciar cuál de los tratamientos generó una diferencia en esta variable, dado que la prueba global no mostró diferencias significativas de los tratamientos frente a las demás variables.

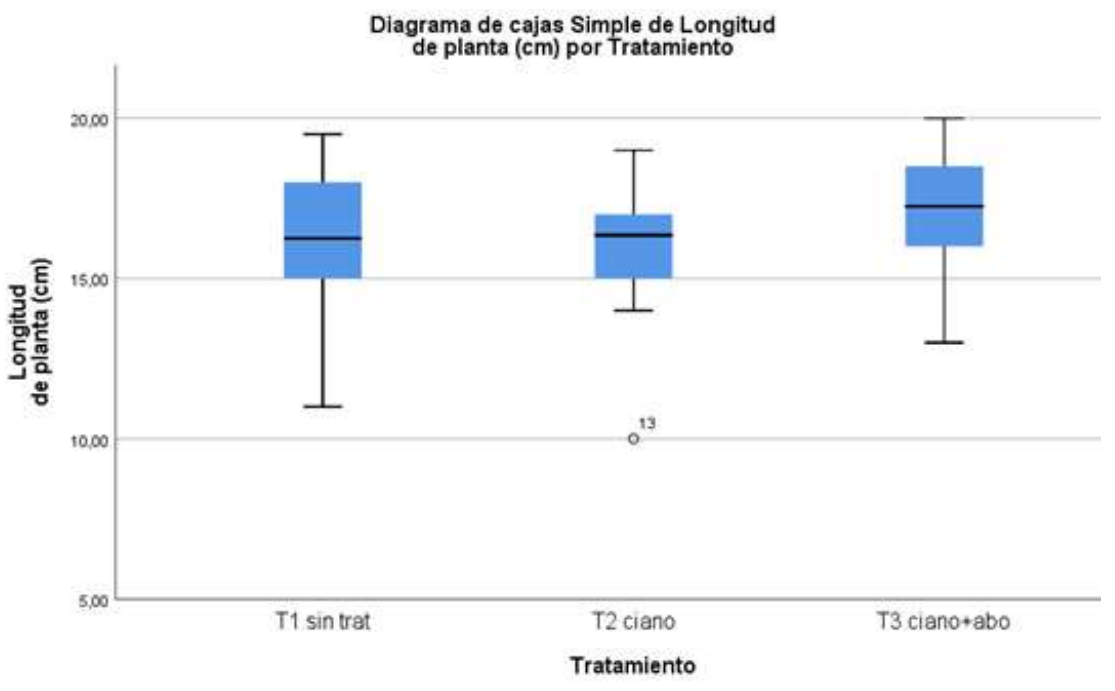

Figura 3. Distribución de la variable longitud de la planta frente a los tratamientos ( $\mathrm{T} 1, \mathrm{~T} 2$ y $\mathrm{T} 3$ ) a lo largo del tiempo de estudio

\section{Discusión}

Con respecto a la longitud de la planta resultados similares se han reportado en estudios anteriores ${ }^{25}$ en uno de ellos se utilizó Azotobacter spp., Nostoc spp. y Anabaena spp. para determinar el crecimiento de plantas de menta (Mentha spicata), y se encontró que las plántulas inoculadas con Azotobacter spp., presentaron un $76 \%$ más de crecimiento, mientras que las plantas tratadas con la cianobacteria Nostoc spp. y el control no mostraron diferencias entre sí con un porcentaje del 59 y $58 \%$. En dicho estudio al igual que en el presente, los resultados obtenidos para la variable LP utilizando diferentes microorganismos como tratamientos se observó que la mayor longitud fue el control del experimento, en el estudio de Ortiz et al., ${ }^{25}$ correspondió a Azotobacter spp. y en el actual al T3. En cuanto a la variable $L R$, se han reportado resultados diferentes. En un estudio se reportó que al inocular Pseudomonas spp. en cultivos de frijol, maíz, papa y tomate, y en el que se evaluó la materia seca de las raíces se observó, en todos los casos, incrementos con relación al control sin inocular, lo que manifestó mayor crecimiento de la parte aérea del cultivo. ${ }^{26}$

En contraste a los resultados obtenidos en el presente estudio, plantas inoculadas con microorganismos pertenecientes al género Bacillus como biofertilizantes en cultivos de tomate y pimiento ${ }^{27}$ presentaron incrementos en el peso fresco de las plántulas en un 17 y un $20 \%$, respectivamente, y un incremento en la biomasa total de planta en 37 y $16 \%$. Las plántulas de tomate se cultivaron por 30 días y las de pimiento por 60 días; caso contrario con los resultados que se obtuvieron para el peso fresco de la raíz donde ninguna cepa tuvo efecto. Estudios anteriores reportaron que el uso las cianobacterias como inoculantes para evaluar el crecimiento de la planta en cultivos de trigo, cuyo tiempo de crecimiento desde siembra hasta cosecha fue de seis meses aproximadamente, dan cuenta del potencial fertilizante de las cianobacterias Calothrix 
ghosei, Hapalosiphon intricatus, y Nostoc spp., al presentar resultados positivos donde se obtuvo una altura máxima de $51,83 \mathrm{~cm}$ en etapa de cosecha bajo condiciones de invernadero, con una frecuencia de inoculación de 15 y 30 días después de la siembra. ${ }^{28}$

Según los resultados obtenidos en el presente estudio, en función del coeficiente de correlación de Spearman y el coeficiente de determinación, la variable LR no presentó un aumento significativo en función del tiempo en el que fue evaluada, mientras que en un estudio reportado por Freire, ${ }^{29}$ donde utilizaron dos consorcios de cianobacterias para evaluar sus efectos sobre la altura de la planta y la longitud de la raíz en pastos raygrass (Lolium multiflorum), se obtuvieron alturas del pasto de 23,16 y 21,18 cm; la longitud de la raíz alcanzó $4,68 \mathrm{~cm}$ de crecimiento, denotando una tendencia positiva durante los 35 días de evaluación del cultivo.

Los resultados que se obtuvieron para la variable PFR se contrastan con los datos obtenidos en el estudio de Himani, ${ }^{30}$ donde se evaluó la influencia de cianobacterias en los parámetros de crecimiento de plantas de arroz, en peso fresco de la planta y la raíz, con 10 días de crecimiento, los resultados de dicho estudio presentaron raíces con un peso de 15,10 mg y $24,70 \mathrm{mg}$ en las plantas control sin inocular e inoculadas, respectivamente. El peso fresco de las plantas de control fue de 76,67 mg, mientras que el de las plantas inoculadas fue cuatro veces mayor con valores de $323,33 \mathrm{mg}^{30}$

Resultados obtenidos en otros estudios, son comparables con los del presente, como por ejemplo el de Araujo et al. ${ }^{31}$ donde se comparó el efecto la de inoculación de cianobacterias en cultivos de arroz, maíz y frijol, frente a tratamientos de fertilización química con Nitrógeno; los resultados obtenidos al evaluar la variable altura de la planta plantean que no existe diferencia significativas entre estos tratamientos al observarse porcentajes de altura de la planta para el tratamiento con cianobacterias que van desde $8,8 \%$ al día 37 y $15 \%$ para el día 74 de evaluación, datos semejantes a los presentados por las plantas tratadas con fertilización química con Nitrógeno. Bidyarani et al. ${ }^{32}$ evaluaron el aumento del crecimiento y el rendimiento de plantas de garbanzo (Cicer arietinum L.) con inoculantes de cianobacterias y rizobacterias durante la etapa media de cultivo (75d) y cosecha (150d), los resultados indicaron que la variable peso seco de la planta alcanzó su valor máximo $(40,53 \mathrm{~g})$, número de máximo de vainas por planta $(87,33)$ y rendimiento de grano $(24-50 \%)$ con los tratamientos basados en cianobacterias como cepas individuales o biopelículas, estos mostraron un mejor rendimiento para la mayoría de los parámetros evaluados, respecto a los otros tratamientos.

Esto resultados y los de otros estudios evidencian que las investigaciones con cianobacterias requieren mayor precisión en el establecimiento de los controles y de los niveles de las variables del estudio, así como también ahondar en las concentraciones de las cianobacterias, las especies empleadas, y las condiciones del medio donde se da el proceso de evaluación de las plantas, entre otros; todos estos aspectos se deben tener en cuenta para posteriores estudios. Se ha reportado que la introducción de cianobacterias puede establecerse de forma permanente y mejorar la calidad del suelo si la inoculación se realiza de manera consecutiva para tres o cuatro ciclos de cultivo. ${ }^{33}$ Adicionalmente, es probable que la concentración de cianobacterias influya directamente sobre el crecimiento de las plantas, como lo demostró Cruz ${ }^{20}$ quien aplicó a sus tratamientos una concentración de $6,9 \times 10^{7}$ células $/ \mathrm{mL}$, lo que le garantiza una etapa metabólica óptima de los microorganismos. Otros estudios realizados en la India donde la estrategia de inoculación de las cianobacterias se llevó a cabo sobre semillas de especias como el comino, demostraron un porcentaje de germinación de 91,67\% al tratar, en particular, con la cianobacteria Anabaena laxa. ${ }^{19}$

En cuanto al tipo de microorganismo seleccionado para la biofertilización se reporta que la mayoría de los ensayos donde se han usado las cianobacterias se formulan con especies que presentan heterocistos, algunas de las más usadas en los biopreparados son: Aulosira fertilissima, Anabaena sphaerica, Nostoc hatei, Cylindrospermum majusand y Westiellopsis prolifica. . $^{10,33-35}$ Gracias a estudios anteriores se conoce que las cianobacterias más frecuentes en el embalse Peñol-Guatapé son Mycrocystis aeruginosa, Synechococcus rubescens y Cyanobium gracile, ${ }^{23}$ no formadoras de heterocistos y no diazotróficas; sin embargo, en un estudio realizado en 2018 por Araujo et al, ${ }^{31}$ se encontró que la inoculación a plantas de arroz con la cianobacteria Gloeocapsa spp., promovió el crecimiento de la planta aunque esta no 
es formadora de heterocisto, lo que sugiere que estas células especializadas en la fijación de nitrógeno no son la única forma de favorecer el crecimiento de las plantas, puesto que existen otras vías como la formación de auxinas, entre otros mecanismos. ${ }^{14}$

Se ha informado que a la hora de inocular los microorganismos en el suelo, estos presentan dificultad para colonizar las raíces y sobrevivir; por lo que resulta de gran importancia tener en cuenta que la supervivencia de las cepas va a depender de la textura, el $\mathrm{pH}$, la temperatura, la humedad y la disponibilidad de nutrientes en el suelo. ${ }^{36,37}$ Por otra parte, al momento de inocular las cianobacterias directamente en el suelo, el contenido de agua de este experimenta variaciones continuas a lo largo del tiempo, como recibir aguas lluvias y perderlas por escorrentía superficial, por drenaje hacia las capas más profundas, y por evapotranspiración. ${ }^{38}$

Finalmente, en la literatura no se encuentran reportes de cianobacterias obtenidas e inoculadas a partir de medios acuosos donde se presentan al mismo tiempo procesos de degradación de materia orgánica. $\mathrm{Si}$ bien es cierto que la presencia de materia orgánica podría generar un sesgo al convertirse en un elemento de fertilización de las plantas tratadas por el contenido de nutrientes, especialmente nitrógeno y fósforo, disueltos en la muestra de agua, esta concentración probablemente no sea significativa. Según Haro y Perales ${ }^{39}$ la eliminación o asimilación de $\mathrm{N}$ y $\mathrm{P}$ por un «bloom» de microalgas comienza casi de inmediato, partiendo de concentraciones muy por encima (14 mg $\mathrm{N}$ total soluble/L y $1.9 \mathrm{mg}$ P total soluble/L) de las registradas en estudios previos en la muestra de agua utilizada. Lo anterior evidencia una de las ventajas de este tipo de estudios, pues permiten emplear aguas eutrofizadas con "bloom" para la fertilización de cultivos, permitiendo de esta manera no solo su utilización sino la oportunidad de darle manejo a fuentes de aguas contaminadas con materia orgánica.

\section{Conclusiones}

Los resultados evidenciaron que no hubo diferencias entre tratamientos; sin embargo, es interesante que un tratamiento no convencional presente resultados similares a un tratamiento orgánico que conlleva costos de manejo y certificaciones que hacen más difícil su implementación.

En la actualidad se han desarrollado estudios en diversos tipos de cultivos, principalmente en cereales y leguminosas utilizando cianobacterias como biofertilizantes. No obstante, es necesario ahondar en estudios que evalúen el potencial de biofertilización con cianobacterias en cultivos autóctonos, teniendo conocimiento de los problemas que representan, para el suelo y su ecología, el uso de fertilizantes de origen químico.

\section{Conflictos de interés}

Los autores declaran no tener ningún conflicto de interés.

\section{Referencias}

1. Creus CM. Microbial inoculants: Pieces of a puzzle that still needs to be assembled. Rev Argent Microbiol. 2017;5(1):207-209.

2. Youssef MMA, Eissa MFM. Biofertilizers and their role in management of plant parasitic nematodes. A review. J Biotechnol Pharmaceut Research. 2014;5(1):001-006.

3. Binder C, García G, Andreoli R, Diaz J, Feola G, Wittensöldner $\mathbf{M}$, et al. Simulating Human and Environmental Exposure from Hand-held Knapsack Pesticide Application: Be-wetspa-pest, An Integrative, SpatiaIly Explicit Modeling Approach. J Agric Food Chem. 2016;64(20):3999-4008.

4. Instituto Nacional de Salud [INS]. Vigilancia y análisis del riesgo en salud pública. Protocolo de vigilancia en salud pública. Intoxicaciones por sustancias químicas. Bogotá D.C: Secretaria de Salud; 2016.

5. Organización Mundial de la Salud [OMS]. Más medidas para mejorar la inocuidad de los alimentos. Ginebra: OMS; 2017.

6. Medina N, León O. Biotechnology and sustainable agriculture: biofertilizers and biopesticides. Natural Institute of Agricultural Science. La Habana: Pugwash Workshop; 2004.

7. Ghosh N. Promoting bio-fertilizers in Indian agriculture. Institute of Economic Growth. Delhi: University Enclave; 2002.

8. Prasanna R, Jaiswal $P$, Singh $Y$, Singh $P$. Influence of biofertilizers and organic amendments on nitrogenase activity and phototrophic biomass of soil under wheat. Acta Agronomica Hungarica. 2008;56(2):149-159. 
9. Vaishampayan A, Sinha RP, Hader DP, Dey T, Gupta AK, Bhan U, et al. 2001. Cyanobacterial biofertilizers in rice agriculture. Botanical Rev. 2001;67: 453-516.

10. Rodríguez AA, Stella AM, Storni MM, Zulpa G, Zaccaro MC. Effects of cyanobacterial extracellular products and gibberellic acid on salinity tolerance in Oryza sativa L. Saline Systems. 2006;2:7.

11. Rai AK, Sharma NK. Phosphate metabolism in the cyanobacterium anabaena doliolum under salt stress. Current Microbiology. 2006;52:6-12.

12. Stihl A, Sommer U, Post AF. Alkaline phosphatase activities among populations of the colonyforming diazotrophic cyanobacterium Trichodesmium spp. (cyanobacteria) in the Red Sea. J Phycology. 2001;37(2):310-317.

13. Rastogi RP, Sinha RP. Biotechnological and Industrial Significance of Cyanobacterial Secondary Metabolites. Biotechnol Adv. 2009;27(4): 521-539.

14. Sharma NK, Tiwari SP, Tripathi K, Rai AK. Sustainability and cyanobacteria (Blue-green Algae): Facts and challenges. J Appl Phycology. 2001; 23:1059-1081.

15. Ranjan K, Priya H, Ramakrishnan B, Prasanna R, Venkatachalam S, Thapa S, et al. Cyanobacterial inoculation modifies the rhizosphere microbiome of rice planted to a tropical alluvial soil. Amsterdam. Appl Soil Ecol. 2016;108:195-203.

16. Shariatmadari Z, Riahi H, Hashtroudi MS, Ghassempour A, Aghashariatmadary Z. Plant growth promoting cyanobacteria and their distribution in terrestrial habitats of Iran. Soil Sci Plant Nutrition. 2013;59(4):535547.

17. Vessey JK. Plant growth promoting rhizobacteria as biofertilizers. Plant Soil. 2003;255:571-586.

18. Ocampo J, Wyckhuys K. Tecnología para el Cultivo de la gulupa en Colombia (Passiflora edulis $f$. edulis Sims) purple passion fruit. Centro de Bio-sistemas de la Universidad Jorge Tadeo Lozano, Bogotá: Centro Internacional de Agricultura Tropical - Ciat y Ministerio de Agricultura y Desarrollo Rural. Colombia; 2012.

19. Kumar M, Prasanna R, Bidyarania N, Babu S, Kumar Mishra A, Kumar A, et al. Evaluating the plant growth promoting ability of thermotolerant bacteria and cyanobacteria and their interactions with seed spice crops. Scientia Horticulturae. 2013;164(17):94-101.

20. Cruz SMA. Uso biofertilizante de dos cultivos de cianobacterias, uno axénico y otro en consorcio, a nivel de invernadero para producción parcialmente orgánica de fréjol Phaseolus vulgaris. (tesis de pregrado). Ecuador: Universidad de las Fuerzas Armadas; 2009.

21. Aguirre N, Palacio J, Ramírez JJ. Características limnológicas del embalse el Peñol-Guatapé, Colombia. Rev Ing Univ Medellín 2007;6(10):53-66.

22. Hernández CA. Análisis ambiental de las grandes centrales hidroeléctricas de colombia aplicando metodo- logía multiobjetivo. (tesis de pregrado). Colombia: Universidad de la Salle; 2011.

23. Múnera L. Identificación y cuantificación de cianobacterias planctónicas en los embalses Abreo malpaso, Peñol-Guatapé y playas del oriente antioqueño empleando técnicas moleculares. (Tesis de maestría). Medellín: Universidad de Antioquia; 2017.

24. Corporación Autónoma Regional de las Cuencas de los Ríos Negro - Nare [CORNARE] (2012). Evaluación y zonificación de riesgos por avenida torrencial, inundación y movimiento en masa y dimensionamiento de procesos erosivos en el municipio de Marinilla. Marinilla: Gobernación de Antioquia; 2012.

25. Ortiz G. Evaluación del valor como biofertilizantes de Nostoc sp. y Anabaena sp. en plántulas de menta (Mentha spicata). (Tesis de pregrado). Bogotá: Universidad Santo Tomás: Universidad Santo Tomás; 2014.

26. Santillana N, Arellano C, Zúñiga D. Capacidad del rhizobium de promover el crecimiento en plantas de tomate (Lycopersicum esculentum Miller). Ecol Aplicada. 2005;4(1,2):47-51.

27. Luna M, Martínez P, Hernández M, Arvizu S. Pacheco J. Caracterización de rizobacterias aisladas de tomate y su efecto en el crecimiento de tomate y pimiento. Rev Fitotecnia Mex. 2013;36(1):63-69.

28. Karthikeyan N, Prasanna R, Nain L, Kaushik B. Evaluating the potential of plant growth promoting cyanobacteria as inoculants for wheat. Eur J Soil Biol. 2007;43(1):23-30.

29. Freire E, Koch A, Salvador L. Evaluación del potencial biofertilizante de consorcios de cianobacterias en pasto raygrass (Lolium multiflorum). Rev Científica Ecuat. Especial de Suelos. 2016;4(1):13-19.

30. Priya H, Prasanna R, Ramakrishnan B, Bidyarani N, Babu S, Thapa S. et al. Influence of cyanobacterial inoculation on the culturable microbiome and growth of rice. Microbiological Res. 2015;171:78-89.

31. Araujo D, Hernández R, Vanegas J. Efecto de la inoculación de cianobacterias en cultivos de interés comercial en zonas semiáridas de La Guajira. Rev Col Invest Agroindustriales. 2018;5(1):20-31.

32. Bidyarani N, Prassana R, Babu S, Hossain F, Kumar Saxena A. Enhancement of plant growth and yields in Chickpea (Cicer arietinum L.) through novel cyanobacterial and biofilmed inoculants. Microbiological Res. 2016;188-189:97-105.

33. Prasanna R, Joshi M, Rana A, Singh Shivay Y, Nain L. Influence of co-inoculation of bacteria-cyanobacteria on crop yield and $\mathrm{C}-\mathrm{N}$ sequestration in soil under rice crop. World J Microbiol Biotechnol. 2012; 28(3):12231235.

34. Nanjappan K, Prasanna R, Nain L, Kaushik B. Evaluating the potential of plant growth promoting cyanobacteria as inoculants for wheat. Eur J Soil Biol. 2007;43(1):23-30. 
35. Pereira I, Ortega R, Barrientos L, Moya M, Reyes G, Kramm V. Development of a biofertilizer based on filamentous nitrogen-fixing cyanobacteria for rice crops in Chile. J Appl Phycology. 2009;21(1):135-144.

36. Seyhaneyildiz Can S, Koru E, Cirik S. Effect of temperature and nitrogen concentration on the growth and lipid content of Spirulina platensis and biodiesel production. Aquaculture Internatl. 2017;25(5):1-9.

37. Jiménez Y, Carranza C, Rodríguez M. 2009. Manejo Integrado de la gulupa (Passiflora edulis Sims). En: Miranda D, Fischer G, Carranza C., Magnitskiy S, Casierra F,
Piedrahíta W, et al. (eds.). Cultivo, poscosecha y comercialización de las pasifloráceas en Colombia: maracuyá, granadilla, gulupa y curuba. Sociedad Colombiana de Ciencias Hortícolas. 2009;121-158.

38. Angella G, Frías C, Salgado R. Conceptos básicos de las relaciones agua-suelo-planta. Argentina: Inta; 2016.

39. Haro S, Perales JA. Cinética del consumo de nutrientes y crecimiento de un bloom de microalgas en un fotobiorreactor High Rate Algae Pond (HRAP). Tecnol Cienc Agua. 2015;7(1):15-31. 